\title{
Procedimiento para la definición y jerarquización de prioridades competitivas de fabricación. Aplicaciones en las pymes de la industria metalmecánica
}

\author{
William A. Sarache-Castro*\$, Diana M. Cárdenas-Aguirre*, Jaime A. Giraldo* \\ * Departamento de Ingeniería Industrial de la Universidad Nacional de Colombia, Sede Manizales. \\ §e-mail:wasarachec@unal.edu.co
}

(Recibido: Septiembre 29 de 2005 - Aceptado: Noviembre 29 de 2005)

\begin{abstract}
Resumen
Uno de los problemas fundamentales en el diseño de una estrategia de producción/operaciones se centra en la definición y jerarquización de las prioridades competitivas, que deben orientar las acciones estratégicas para el mejoramiento del sistema productivo. Por tal razón, el presente artículo expone un procedimiento basado en técnicas multicriterio y métodos de expertos, que sirve como herramienta para establecer el conjunto de tareas de fabricación y para apoyar el proceso de construcción de la estrategia de producción.
\end{abstract}

Palabras Clave: Estrategia de Producción/Operaciones, Prioridades Competitivas.

\begin{abstract}
One of the main problems in the design of a production and operations strategy, is the definition and organization by hierarchy of the competitive priorities that should drive the strategic actions to improve the production system. In this sense, the present article shows a procedure, based on multicriteria techniques and experts methods, that serves as a tool to establish the production tasks and to support the construction of the production strategy.
\end{abstract}

Keywords: Operations strategy, competitive priorities.

\section{Introducción}

Actualmente, dado el dinamismo y la incertidumbre del entorno económico mundial, resulta de vital importancia prestar mayor atención a la función de producción/operaciones, concibiéndola como una fuente de creación de ventajas competitivas para la empresa. En este sentido, las compañías de primer orden en la fabricación, buscan la excelencia con mejores procesos y tecnologías de producción e intentan competir simultáneamente en todas las dimensiones de competitividad. Sus formidables organizaciones de fabricación característicamente han desarrollado una filosofía corporativa que sostiene una inteligible cultura de la productividad que perfila y guía su estrategia competitiva. (Dangayach G.S. \& Deshmukh, 2001). 
El proceso estratégico, para la función de producción/operaciones, consta esencialmente de dos fases; en primer lugar, se establecen las prioridades competitivas que orientarán y dirigirán a la producción, requiriéndose una coherencia total entre éstas y la estrategia de negocio; en segundo lugar, y como consecuencia de lo anterior, se lleva a cabo el diseño o el rediseño del sistema de producción, formulándose y poniéndose en práctica un conjunto de políticas de fabricación relativas a las diferentes áreas estratégicas de decisión (Fernández, 2003).

En referencia específica a las prioridades competitivas, Leong et. al. (1990), las definen como un "...conjunto concreto de objetivos o metas para la manufactura". Una revisión cronológica de la literatura ${ }^{1}$, revela que a lo largo del tiempo han surgido nuevas prioridades competitivas para fabricación. El estado del arte, permite constatar la existencia de 7 prioridades competitivas básicas en producción: costos, calidad, entregas, flexibilidad, servicio, innovación y responsabilidad ambiental (Fernández, 2003; Dangayach G.S. \& Deshmukh, 2001). En este punto, es necesario aclarar que en la literatura especializada se han utilizado diversas denominaciones, tales como tareas de producción (Skinner, 1974), metas de fabricación (Miller \& Roth, 1988), objetivos de producción (Anderson et. al., 1989), prioridades competitivas (Leong et. al.,1990), entre otras. Para el caso de la presente contribución, se prefiere usar el término prioridades competitivas.

Las prioridades competitivas señalan la orientación dominante de la producción. El resultado es lo que Skinner (1985) denominó la tarea de producción, donde se pone de manifiesto qué es lo que la función de producción debe hacer bien para el éxito de la estrategia. Sin embargo, para que las prioridades competitivas sirvan de guía para la fijación de políticas en las distintas áreas de decisión, así como para la toma de decisiones a largo y corto plazo, es conveniente su disgregación cualitativa (Garvin, 1994), así como su expresión en términos cuantitativos y mensurables (Schroeder, 1992; Fernández Sánchez, 1993).
Los aportes existentes proponen entonces que, para que una organización productiva pueda desplegar un conjunto de acciones estratégicas competitivas en la función de producción/operaciones, es necesario actuar en tres aspectos fundamentales: 1) la definición de un conjunto de prioridades competitivas relevantes; 2) la jerarquización del conjunto de prioridades estableciendo aquellas más relevantes que deben orientar el direccionamiento estratégico del sistema productivo y 3) la medición integral de su desempeño organizativo en función de las prioridades competitivas seleccionadas. A partir de los resultados, la organización podrá determinar las prioridades en las que se debe concentrar y desplegar las acciones de mejora en el sistema de producción/operaciones necesarias para tales fines. (Vereecke \& Van Dierdonk, 2002).

Lamentablemente, y en referencia al contexto latinoamericano, fuertemente dominado por pequeñas y medianas empresas, la falta de planeación estratégica y las acciones que de ésta se derivan, emergen como una de sus grandes debilidades gerenciales, que a su vez, se reflejan en un conjunto amplio de debilidades estructurales e infraestructurales, no sólo a nivel de la función de producción/operaciones, sino de otras áreas fundamentales. (Barriga Manrique, 1998; Ceglie \& Dini, 1999; CEPAL, 2001).

Los estudios en el tema han recalcado un conjunto de debilidades organizativas en las PyME's que, en materia de gestión de la producción/operaciones, se pueden resumir en las siguientes: deficiencias en la calidad, baja fiabilidad en las entregas, limitaciones en la capacidad de fabricación, dificultades para establecer costos de producción, baja capacidad de innovación y bajos niveles de desempeño en servicio. Sin embargo, se reconoce que a nivel nacional el tema estrategia de operaciones ha sido poco abordado desde el punto de vista investigativo y por tanto, las contribuciones sobre el mismo son escasas. No obstante, las contribuciones internacionales han sido prolíficas, generando importantes aportes en sectores industriales que sobreviven en entornos turbulentos.

Por tal razón, como contribución al área de estudio, el presente artículo presenta los resultados del diseño y aplicación de un procedimiento para la selección de 
prioridades competitivas y su jerariquización, apoyada en la aplicación de métodos de expertos y técnicas de ponderación multicriterial. Se propone, así mismo, la construcción de un indicador de efectividad para medir de manera integral el grado de acercamiento de una organización o sector al nivel de excelencia en un conjunto de prioridades. Finalmente se presentan, para efectos de su aplicación, los resultados en el sector metalmecánico de la ciudad de Manizales.

Dicho procedimiento, constituye una herramienta práctica que puede ser aplicada a nivel de una organización o de un sector empresarial. Éste, forma parte de los resultados de la investigación intitulada "Modelo, procedimientos y soluciones informáticas para mejorar el nivel de efectividad de los sistemas de producción de PyME's metalmecánicas. Aplicaciones en el subsector 381 de la ciudad de Manizales", la cual se encuentra en curso y cuenta con la financiación de COLCIENCIAS y la Dirección de Investigaciones (DIMA) de la Universidad Nacional de Colombia, Sede Manizales.

\section{Desarrollo del procedimiento}

El procedimiento propuesto está compuesto por 7 pasos, tal como se expone en la Figura 1. Su construcción se hizo retomando algunos aportes de Boxwell (1995) y Christopher (1994), enriquecido con los aportes de Romero (1993), Barba-Romero \& Pomerol (1997), Marrero Delgado (2001) y Calves Hernández \& Calderón Millian (1998), mediante la introducción y aplicación de métodos de expertos y técnicas multicriterio.

El objetivo general del procedimiento es desarrollar, de una manera sistemática y ordenada, en primer lugar, un proceso de definición y jerarquización de prioridades competitivas de manufactura y en segundo lugar, construir un indicador de efectividad o desempeño que permita soportar procesos de referenciación competitiva (benchmarking interno y benchmarking competitivo). Para ello, el procedimiento se sustenta en los siguientes principios: consistencia lógica, flexibilidad, parsimonia, trascendencia, racionalidad, sistematicidad y perspectiva. Una descripción sintética de las etapas es la siguiente:
Paso 1. Definir las prioridades fundamentales: Este paso consiste en detectar las exigencias de los clientes que conforman el mercado objetivo. Tales

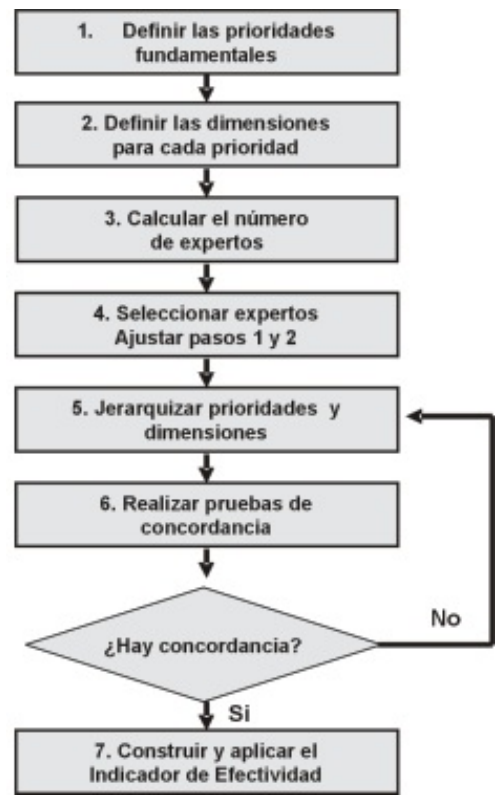

Figura 1. Procedimiento para la selección y jerarquización de prioridades competitivas.

exigencias no son más que las prioridades competitivas para la fabricación. Su detección se puede hacer a través de investigación de mercados, encuestas, estudios de benchmarking, grupos de expertos o valoraciones preliminares a partir de tendencias del mercado o investigaciones académicas previas.

Paso 2. Definir las dimensiones para cada prioridad: para cada prioridad competitiva se debe definir el conjunto de dimensiones que son de relevancia; por ejemplo, para el caso de las entregas, normalmente surgen como dimensiones relevantes el plazo, la fiabilidad en la entrega y la rapidez del sistema de información, entre otras. Su definición se realiza de igual manera que en el paso 1 .

Paso 3. Calcular el número de expertos: Se propone en esta fase del procedimiento, la aplicación de los denominados métodos de expertos. El cálculo del número de expertos se realiza mediante la aplicación de la expresión 1. 
Dónde:

$$
n=\frac{P(1-P) K}{i^{2}}
$$

n: Número de expertos.

i: Nivel de precisión deseado.

p: Porcentaje de error que como promedio se tolera.

$\mathrm{k}$ : Constante asociada al nivel de confianza.

Paso 4. Seleccionar expertos y ajustar pasos 1 y 2 : "los expertos seleccionados deben ser tales que sus motivaciones e intereses no se superpongan con el problema que deben abordar" (Calvés Hernández \& Calderón Millian, 1998). En este sentido, los expertos pueden ser los propios clientes del mercado objetivo, sus representantes (para el caso de clientes corporativos) o expertos conocedores del mercado objetivo (gremios, académicos, agencias gubernamentales, etc). Finalmente, se procede a depurar la lista preliminar de prioridades y sus dimensiones, hasta obtener una lista definitiva libre de redundancias.

Paso 5. Jerarquizar prioridades y dimensiones: En este paso, los expertos deben proceder a "calificar" la importancia relativa entre el conjunto de prioridades y entre las dimensiones de cada prioridad. Para su desarrollo se pueden aplicar métodos de jeraquización simple o más avanzados como el método de Saaty (Barba-Romero \& Pomerol, 1997). El resultado final es la obtención del orden de importancia relativa entre prioridades y entre dimensiones.

Paso 6. Realizar pruebas de concordancia: La prueba consiste en medir el nivel de acuerdo entre los expertos a través del indicador de concordancia de Kendall (W). En cada caso, si W es mayor o igual a 0.5 , se considera que existe un buen nivel de acuerdo y por tanto, las ponderaciones se validan (Siegel, 1994). El subprocedimiento a seguir se expone a continuación:

1) Calcular el valor medio de los rangos $(\mathrm{T})$.

$$
T=\frac{1}{2} M(C+1)
$$

2) Calcular la desviación del valor medio de los juicios emitidos para cada criterio $\left(\mathrm{D}_{\mathrm{i}}^{2}\right)$.

$$
D_{i}^{2}=\left[\sum_{j=1}^{M} A i j-T\right]^{2}
$$

3) Calcular el coeficiente de concordancia de Kendall (W).

$$
W=\frac{12 \sum D^{2}{ }_{i}}{M^{2}\left(C^{3}-C\right)}
$$

Donde:

T: Factor de comparación (valor medio de los rangos).

M: Número de expertos.

C: Número de criterios (prioridades o dimensiones).

$\mathrm{D}_{\mathrm{i}}^{2}$ :Desviación al cuadrado del valor medio para cada criterio.

$\mathrm{A}_{\mathrm{ij}}$ : Orden de Importancia dada al criterio i por el experto j.

W: Coeficiente de Concordancia de Kendall.

Finalmente se calcula la ponderación de cada criterio.

Paso 7. Construir y aplicar el Indicador de Efectividad: A partir de las ponderaciones validadas, se procede a la construcción del indicador de efectividad, el cual permite, de manera integral, medir el grado de acercamiento de la organización a los niveles de excelencia en las prioridades competitivas y dimensiones seleccionadas. $\mathrm{Su}$ estructura particular depende del número de prioridades y del número de dimensiones por cada prioridad. Un modelo de la estructura general del indicador es el que se propone en la expresión 5.

$$
I E i=\left[\sum_{j=1}^{n} W i j\left(\sum_{k=1}^{l} W i j k \bullet C i j k\right)\right]
$$

Donde:

$\mathrm{IE}_{\mathrm{i}}$ : Indicador de Efectividad que alcanza una empresa i.

$\mathrm{W}_{\mathrm{ij}}$ : Peso de la prioridad $\mathrm{j}$ en la empresa $\mathrm{i}$ (calidad, precio, entrega, flexibilidad, servicio e 
innovación).

$\mathrm{W}_{\mathrm{ij \textrm {k }}}:$ Peso de la dimensión $\mathrm{k}$ en el factor $\mathrm{j}$ para la empresa i.

$\mathrm{C}_{\mathrm{ijk}}$ : Calificación obtenida por la dimensión $\mathrm{k}$, en la prioridad $j$, para la empresa $i$.

\section{Resultados de aplicación}

Para efectos de aplicar el procedimiento, se seleccionó un grupo de empresas del sector metalmecánico de la ciudad de Manizales. El procedimiento general que se siguió fue el siguiente: (1) construcción del indicador de desempeño, (2) construcción del instrumento de recolección de datos, (3) detección del Universo, (4) cálculo del tamaño de muestra y selección de las empresas y (5) aplicación del instrumento de recolección de datos y del IEi. Una síntesis de los resultados obtenidos es la siguiente:

Pasos 1 al 4: Se construyó una lista preliminar de prioridades y dimensiones a partir de las tendencias del estado del arte y consultas a expertos. El listado preliminar se elaboró a partir de exigencias mundiales. Seguidamente y aplicando la expresión 1, se obtuvo un cálculo de 9.23 expertos, es decir, 10. Los valores utilizados para la expresión 1 fueron:

$$
\mathrm{P}=0.03 ; \mathrm{i}= \pm 0.11 ; \mathrm{K}=3,8416 \text { para } 1-=9.23 \text {. }
$$

Los expertos seleccionados fueron gerentes de empresas con vocación exportadora, directivos gremiales y expertos en mercados internacionales. Por último, los expertos procedieron a depurar la lista preliminar, eliminando prioridades y dimensiones no relevantes o redundantes. El listado definitivo se expone en la tabla 1.

Pasos 5 y 6: Dadas las dificultades y exigencias de presencialidad que le subyacen a los métodos avanzados, para el presente caso se prefirió aplicar una técnica de jerarquización simple. Una ilustración del resultado obtenido para el conjunto de prioridades se expone en la tabla 2. Al aplicar las expresiones de cálculo 2, 3 y 4, para la tabla 2 , los resultados son:

$$
\mathrm{T}=35 ; \mathrm{D}^{2}{ }_{\mathrm{i}}=963.5 ; \mathrm{W}=0.55 \text {. }
$$

\begin{tabular}{|c|c|}
\hline $\begin{array}{l}\text { Prioridades } \\
\text { competitivas } \\
\text { j }\end{array}$ & $\begin{array}{l}\text { Dimensiones } \\
(D j k)\end{array}$ \\
\hline \multirow[t]{3}{*}{ Precio } & Sin dimensiones \\
\hline & $\begin{array}{l}\text { Calidad de concepción: Diseños basados en las característic as solicitadas por los } \\
\text { clientes. }\end{array}$ \\
\hline & $\begin{array}{l}\text { Calidad de concordancia: Productos acordes con las especificaciones de diseño. } \\
\text { Durabilidad del producto: Lapso de tiem po en el que el producto está en } \\
\text { condiciones apropiadas de operación. }\end{array}$ \\
\hline \multirow{2}{*}{ Calidad } & $\begin{array}{l}\text { Fiabilidad del producto: Probabilidad de prestar un servicio adecuado en un periodo } \\
\text { de tiempo determinado. }\end{array}$ \\
\hline & $\begin{array}{l}\text { Plazo: Tiempo que transcurre desde el momento en que se recibe una } \\
\text { orden de pedido hasta que éste es entregado al cliente. }\end{array}$ \\
\hline \multirow[t]{2}{*}{ Entrega } & $\begin{array}{l}\text { Fiabilidad en la entrega: P orcentaje de entregas realizadas en el mom ento y la } \\
\text { cantidad pactados. }\end{array}$ \\
\hline & $\begin{array}{l}\text { Información oportuna sobre el estado de av ance de los pedidos. } \\
\text { Flexibilidad en el tamaño de lote: Capacidad para fabricar lotes de cualquier } \\
\text { tamaño. }\end{array}$ \\
\hline \multirow[t]{3}{*}{ Flexibilidad } & $\begin{array}{l}\text { Flexibilidad ant e cambios imprevi stos: Capacidad para asimilar cam bios no } \\
\text { programados. }\end{array}$ \\
\hline & Flexibilidad en produc tos: capacidad para ofertar una ampl ia gama de productos. \\
\hline & $\begin{array}{l}\text { Agilidad para responder a la solicit ud de cotización del client e. } \\
\text { Garantía y Servici o post-venta. }\end{array}$ \\
\hline \multirow[t]{2}{*}{ Servicio } & Facilidades para realizar dev olución. \\
\hline & \\
\hline & Desarrollo de productos altamente di ferenciados en el mercado. \\
\hline
\end{tabular}

Tabla 1. Listado final de prioridades competitivas y dimensiones

De la misma manera se procedió para las dimensiones de la calidad, la entrega, la flexibilidad, el servicio y la innovación.

La Tabla 3 presenta una síntesis de la jerarquización (ponderación) final obtenida. Los resultados destacan la importancia que, para los expertos consultados, presentan la calidad y el plazo, inclusive, por encima del precio. Por otro lado, al valorar la ponderación de prioridades y dimensiones, es claro que para el sector en estudio resulta vital orientar sus acciones estratégicas hacia la reducción de plazos, fortalecimiento de la calidad y aumento de la flexibilidad en productos y programas.

Tabla 2. Jerarquización (ponderación) de prioridades competitivas

\begin{tabular}{cccccccc}
\hline Experto & PRECIO & CALIOAD & ENTREGA & FLEXIBIIDAD & SERVICIO & INNOVACIÓN & TOTALES \\
\hline 1 & 6,0 & 3,0 & 5,0 & 1,0 & 4,0 & 2,0 & 21 \\
2 & 4,0 & 6,0 & 5,0 & 1,5 & 3,0 & 1,5 & 21 \\
3 & 1,5 & 6,0 & 5,0 & 1,5 & 4,0 & 3,0 & 21 \\
4 & 6,0 & 4,0 & 1,0 & 2,0 & 3,0 & 5,0 & 21 \\
5 & 4,0 & 6,0 & 5,0 & 1,0 & 2,0 & 3,0 & 21 \\
6 & 4,0 & 6,0 & 5,0 & 3,0 & 2,0 & 1,0 & 21 \\
7 & 4,0 & 6,0 & 5,0 & 1,0 & 3,0 & 2,0 & 21 \\
8 & 1,0 & 6,0 & 5,0 & 2,0 & 4,0 & 3,0 & 21 \\
9 & 4,0 & 5,0 & 6,0 & 3,0 & 2,0 & 1,0 & 21 \\
10 & 2,5 & 6,0 & 5,0 & 2,5 & 4,0 & 1,0 & 21 \\
TOTAL & 37,0 & 54,0 & $\mathbf{4 7 , 0}$ & 18,5 & 31,0 & $\mathbf{2 2 , 5}$ & 210,0 \\
\hline PONDERACION & $\mathbf{0 , 1 8}$ & $\mathbf{0 , 2 6}$ & $\mathbf{0 , 2 2}$ & $\mathbf{0 , 0 9}$ & $\mathbf{0 , 1 5}$ & $\mathbf{0 , 1 1}$ & $\mathbf{1}$
\end{tabular}

Tabla 3. Jerarquización de prioridades y dimensiones

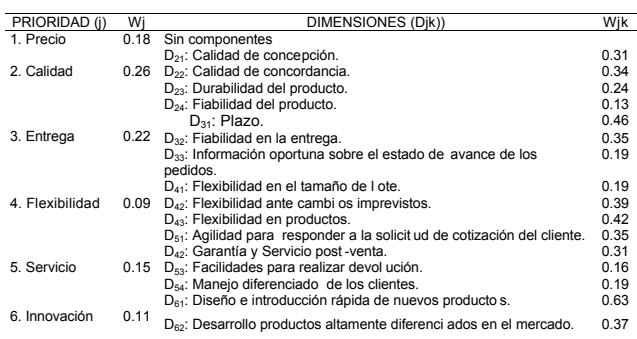


Paso 7: El indicador particular obtenido, para medir el nivel de desempeño de las empresas objeto de estudio, es el siguiente:

$$
\begin{aligned}
& \mathbf{I E}_{\mathrm{i}}=0.18\left[\mathrm{C}_{\mathrm{i} 1}\right]+0.26\left[0.31 \mathrm{C}_{\mathrm{i} 21}+0.34 \mathrm{C}_{\mathrm{i} 22}+0.24 \mathrm{C}_{\mathrm{i} 23}+0.13\right. \\
& \left.\mathrm{C}_{\mathrm{i} 24}\right]+0.22\left[0.46 \mathrm{C}_{\mathrm{i} 31}+0.35 \mathrm{C}_{\mathrm{i} 32}+0.19 \mathrm{C}_{\mathrm{i} 33}\right]+\left[0.19 \mathrm{C}_{\mathrm{i} 41}+\right. \\
& \left.0.39 \mathrm{C}_{\mathrm{i} 42}+0.42 \mathrm{C}_{\mathrm{i} 43}\right]+0.15\left[0.35 \mathrm{C}_{\mathrm{i} 51}+0.31 \mathrm{C}_{\mathrm{i} 52}+0.16 \mathrm{C}_{\mathrm{i} 53}+\right. \\
& \left.0.19 \mathrm{C}_{\mathrm{i} 54}\right]+0.11\left[0.46 \mathrm{C}_{\mathrm{i} 61}+0.35 \mathrm{C}_{\mathrm{i} 62}+0.19 \mathrm{C}_{\mathrm{i} 63}\right] .
\end{aligned}
$$

El resultado final de la aplicación del IEi para las 15 empresas, en una escala de 5 puntos, se resume en la tabla 4

Tabla 4. Indicador de efectividad IEi para las empresas objeto de estudio

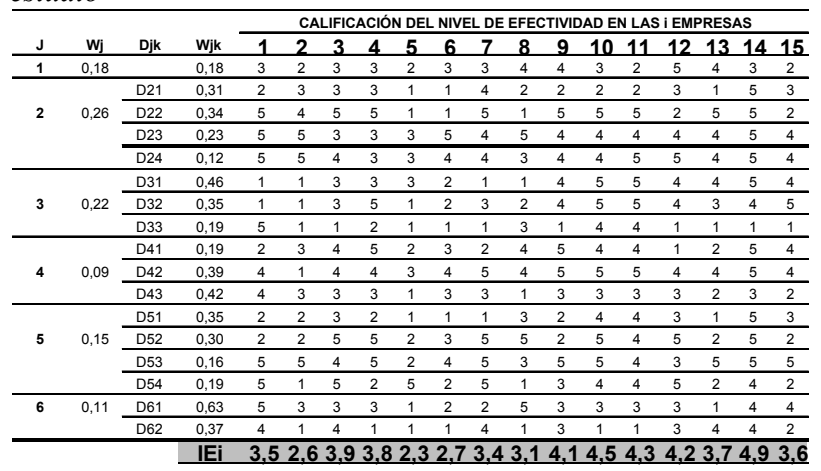

Los resultados obtenidos presentan el nivel de efectividad o de acercamiento de las empresas estudiadas en las 6 prioridades competitivas. En este caso, resulta crítico el desempeño de las empresas 2, 5 y 6. Por otro lado, el desempeño de 4.9 obtenido por la empresa 14, merece ser revisado a la luz de sus verdaderas capacidades de fabricación, toda vez que el instrumento que se utilizó se manejó a manera de autoevaluación. La práctica ha demostrado que difícilmente una empresa pequeña alcanza desempeños altos en todas las prioridades y dimensiones.

\section{Conclusiones y recomendaciones}

Actualmente, para que una organización productiva pueda mantenerse en altos niveles de desempeño frente a su mercado objetivo, debe mejorar simultáneamente en un conjunto amplio de prioridades competitivas; el estado del arte revela que, en el pasado, a medida que las empresas lograron altos estandares en costo y calidad, surgieron nuevas exigencias asociadas a las entregas (competencia basada en el tiempo), el servicio, la innovación y la responsabilidad ambiental.

En este sentido, el procedimiento propuesto en la presente contribución permite establecer, a través de un conjunto ordenado de pasos, el listado de prioridades relevante para una empresa o un sector y su jerarquía u orden de importancia. Dicha jerarquía permite definir las tareas de fabricación sobre las cuales se debe diseñar el direccionamiento estratégico del sistema productivo o el sistema de operaciones (para el caso de las empresas de servicios).

El procedimiento, que aún es una herramienta en construcción, es susceptible de ser mejorado, a través de la introducción de técnicas más avanzadas y subprocedimientos que enriquezcan cada una de sus etapas; también es susceptible de adaptarse para cualquier sector productivo o empresa, con el fin de establecer, a manera de estudio de benchamarking competitivo, el nivel de desempeño en un conjunto de variables estratégicas.

En relación al sector metalmecánico, los resultados revelan que las entregas, la calidad y el precio (costo), son las tres prioridades competitivas fundamentales sobre las que se debe orientar el sistema de fabricación. Sin embargo, al observar desagregadamente las ponderaciones obtenidas en algunas dimensiones, resalta la necesidad de trabajar en la reducción de plazos de entrega, aumento de la tasa de entregas exitosas (pedidos entregados completos y a tiempo), a la vez que se mejoran los niveles de flexibilidad (capacidad para cambiar ante exigencias no previstas) y las habilidades innovativas en el diseño de productos (lanzamiento rápido de nuevos productos). Es decir, según los expertos, el mercado exige que las empresas mantengan precios competitivos, reduzcan plazos y ofrezcan altos niveles de calidad y de flexibilidad en sus operaciones.

Esto, sin lugar a dudas, exige la adopción de sistemas de producción y de logística integrados, eficaces, con niveles de estandarización adecuados y sistemas de programación y control de la producción 
que faciliten el cumplimiento y la admnistración de las entregas de manera exitosa. En este sentido, el desarrollo e implantanción de sistemas de producción híbridos, la integración de operaciones en cadenas de abastecimiento, la adopción de sistemas robustos de calidad, la implantación de tecnologías de información y comunicación y el desarrollo de competencias laborales adecuadas en el recurso humano, son una condición ineludible para aspirar a mercados de alta exigencia.

Para el caso de las 15 empresas estudiadas, aunque los niveles de desempeño en precio (costo) y calidad son aceptables y en algunos casos sobresalientes, es indiscutible que una buena parte de ellas poseen debilidades en las entregas, en la flexibilidad y en la innovación, lo cual las ubica en una situación de debilidad competitiva frente a las exigencias que, según los expertos, son impuestas por los mercados internacionales. Sin embargo, el resultado sólo indica que para el mercado escogido como objetivo en el presente estudio, algunas de las empresas estudiadas no ofrecen fortalezas competitivas; sin embargo, esto no quiere decir que el desempeño sea igual en un contexto regional o nacional, pues las prioridades y la jerarquía de las mismas pueden cambiar.

\section{Agradecimiento}

Los autores agradecen al Instituto Colombiano para el Desarrollo de la Ciencia y la Tecnología COLCIENCIAS y a la Dirección de Investigaciones de la Universidad Nacional de Colombia, Sede Manizales, por el apoyo financiero a la investigación.

\section{Referencias bibliográficas}

Anderson, J.C.; Cleveland, G. \& Schroeder, R.G. (1989). «Operations Strategy: A Literature Review». Journal of Operations Management, vol. 8, No. 2, pp. 126.

Asone, G. \& Noci, G. (1998): Identifying Effective PMSs for the Deployment of "Green" Manufacturing Strategies. International Journal of Operations \&
Production Management. Vol. 18. No. 4. p. 308-335.

Barba Romero, S. \& Pomerol, J. (1997): Decisiones multicriterio: Fundamentos teóricos y utilización práctica. Universidad de Alcalá, España.

Barriga Manrique (1998): La gestión de la pequeña y mediana industria en los países miembros del grupo andino. Informe global. Documento de Investigación. Universidad EAFIT. Medellín, Colombia.

Boxwell, R.J. (1995): Benchmarking para competir con ventaja. Editorial Mc GrawHill. Madrid, España.

Buffa, E.S. (1984): Meeting the Competitive Challenge. Irwin, Homewood, Illinois.

Calvés Hernández, S. \& Calderón Milian, L. [1998]: Técnicas de trabajo creativo en grupos. Grupo de Estudio de Técnicas de Dirección. Universidad Central "Marta Abreu" de Las Villas. Santa Clara, Cuba

Ceglie, G. \& Dini, M. [1999]: El Desarrollo de Clusters y Redes de PyME's en los Países en Desarrollo: El Caso de Nicaragua. Encuentro, Revista de la Universidad Centroamericana, Año XXXI, No. 50, Nicaragua.

CEPAL (2001): Elementos de competitividad sistémica de las pequeñas y medianas empresas (PyME) del Istmo centroamericano. Informe de investigación para Naciones Unidas. México, D.F

Christopher, M. (1994): Logística y aprovisionamiento. Cómo reducir costes, stocks y mejorar los servicios. Biblioteca de Empresa. Ediciones Folio. Barcelona, España.

Corbett, C \& Wassenhove, L.V. (1993): "Trade-offs? What trade-offs? Competence and Competitiveness in Manufacturing Strategy". California Management Review, vol. 35, p. 107.

Dangayach G.S. \& Deshmukh [2001]: Manufacturing Strategy. Literature Review and Some Issues. International Journal of Operation \& Production Management. Vol 21, No. 7. p. 884-932. En: www.emerald-library.com/ft

De Meyer, A.; Nakane, J.; Miller, J.G \& Ferdows, K. (1989): "Flexibility: the Next Competitive Battle". The Manufacturing Futures Survey. Strategic Management 
Journal, vol. 10, p. 135-144.

Domínguez Machuca J.A., Alvarez Gil, M.J., Domínguez Machuca, M.A., García González, S. \& Ruiz Jiménez, A. (1995): Dirección de Operaciones. Aspectos estratégicos en la producción y los servicios. Editorial Mc-Graw Hill, Madrid, España.

Fernández, E. (2003): Estrategia de Producción. Mc Graw Hill, Madrid.

Fernández Sánchez, E. (1993): Dirección de la Producción. Fundamentos estratégicos. Civitas, Madrid.

Fine, C.H. \& Hax, A.C. (1985): "Manufacturing Strategy: A Methodology and an Illustration". Interfaces, vol. $15, \mathrm{~N}^{\circ} 6$, p. 28-46.

Garvin, D.A. (1994): "Planificación estratégica de la producción”. Harvard Deusto Business Review, N${ }^{\circ} .59$, p. 71-85.

Hayes, R.H.; Wheelwright, S.C. \& Clark, K. (1988): "Dynamic Manufacturing". Free Press, N.York.

Hayes, R.H. \& Schmenner, R.W. (1977): "How Should you Organize Manufacturing?". Harvard Business Review, vol. 55, $\mathrm{N}^{\circ} .1$, p. 105-119.

Hayes, R.H. \& Wheelwright, S.C. (1984): Restoring Our Competitive Edge. John Wiley, New York.

Leong, G.K.; Snyder, D.L. \& Ward, P.T. (1990): "Research in the Process and Content of Manufacturing Strategy". Omega International J. of Management Science, vol. 18 (2), p.109-122.

Marrero Delgado, F. (2001): Procedimientos para la toma de decisiones logísticas con enfoque multicriterio en la cadena de corte, alza y transporte de la caña de azúcar. Aplicaciones en el CAI de la provincia de Villa Clara. Tesis de Doctorado. Universidad Central "Marta Abreu" de Las Villas. Santa Clara, Cuba.

Mayer, R.J \& Moore, J. (1983): “Applying Manufacturing Strategy Concepts to Practice". Operations Management Review, (fall issue), p. 23-28

Miller, J.G. \& Roth, A.V. (1988): "Manufacturing Strategies: Executive Summary of the 1987 North American Manufacturing Futures Survey". Operations Management Review, vol. 6, $\mathrm{n}^{\circ}$ 1, p. 8-20.
Olhager, J. \& West, B.M. (2002): "The House Of Flexibility. Using The QFD approach To Deploy Manufacturing Flexibility". International Journal Of Operations \& Production Management. Vol. 22. No 5. p.50-79.

Romano, J.D. (1983): “Operations Strategy"; en Albert, K. (ed.) Strategy Management. McGraw-Hill.

Romero, C. [1993]: Teoría de la decisión multicriterio: conceptos, técnicas y aplicaciones. Alianza Editorial. Madrid.

Sarkis, J. (2001): "Manufacturing's Role in Corporate Environmental Sustainability. Concerns for the New Millennium". International Journal of Operations \& Production Management. Vol. 21. No. 5/6., p. 666-686.

Schroeder, R.G. (1992). Administración de Operaciones. Toma de decisiones en la función de operaciones. $3^{\mathrm{a}}$ edición. Mc Graw-Hill. México.

Schroeder, R.G. \& Lahr, T.N. (1990): "Development of Manufacturing Strategy: A Proven Process"; en Ettlie, J.E.; Burstein, M.C. \& Fiegenbaum, A. (eds). Manufacturing Strategy. Kluwer Academic Publishers, Boston.

Siegel, S. [1994]: Estadística no paramétrica. Aplicada a las ciencias de la conducta. Editorial Trillas. México, D.F.

Skinner, W. (1974): "The Focused Factory". Harvard Business Review, (may-june), p. 113-121.

Skiner, W (1969) "Manofacturing Missing Link in Corporate Strategy" Harvard Bussines Review, (MayJune), p. 136-145.

Skinner, W. (1985): Manufacturing: The Formidable Competitive Weapon. Wiley \& Sons, New York.

Tunc, E. \& Gupta, J.N. (1993): "Is Time a Competitive Weapon Among Manufacturing Firms?". International Journal of Operations \& Production Management, vol. 13, No. 3, p. 4-12.

Vereecke, A. \& Van Dierdonk, R. [2002]: The Strategic Role of the Plant: Testing Ferdow's Model. International Journal of Operation \& Production Management. Vol 22, No. 5. p. 412-514. En: www.emerald-library.com/ft. 